\title{
Effect of Longstanding Diabetes Mellitus Type II on Hand Grip Strength and Pinch Power of Females in the City of Hail-KSA
}

\author{
Mohamed E. Khallaf Ph D PT*, Eman E. Fayed MSc PT **, \\ Manal I. Al-rashidi BSc PT ${ }^{* *}$ \\ * Department of Physical Therapy for Neuromuscular Disturbances and its Surgery, College of Physical \\ Therapy, Cairo University, Egypt. \\ ** Department of Physical Therapy, College of Applied medical sciences, University of Hail -KSA.
}

\begin{abstract}
Background: Diabetes mellitus type II is the most common endocrine disorder all over the world, which is characterized by metabolic abnormal conditions and many long term illness. These complications can lead to social threatening problems due to loss of jobs. Objectives: This study aimed at measuring the effects of Type II diabetes mellitus on hand grip and Pinch power of adult females in the city of Hail-KSA Methods: A cross-sectional study was carried out in the outpatient clinics of the King Khalid Hospital. Forty Female patient represented the sample of the study. A JAMAR dynamometer and a pinch gauge were used to measure the participants' hand holding action and Pinch power. Results: Statistical analysis showed a significant decrease $(P \leq 0,05)$ of the hand grip and pinch power strength among patient with long standing diabetes as compared to healthy matched group. Conclusion :The results of the current study concluded that the hand muscles strength represented in grip and pinch power which are important parameters of hand function is significantly affected by long standing type 2 diabetes mellitus.

Key Words: Diabetes - Diabetic neuropathy - hand grip strength - pinch power
\end{abstract}

\section{Introduction.}

Diabetes mellitus is a chronic metabolic condition characterized by persistent hyperglycaemia, with resultant morbidity and mortality. Type 2 diabetes mellitus (DMII) accounts for about $95 \%$ of diagnosed diabetes in adults. ${ }^{1}$

It is considered as is one of the most common risk factor for functional disability specially after a long duration of illness in older people. It may be associated with peripheral neuropathy, coronary, cerebral or tangential vascular disease, retinopathy, nephropathy, diabetic foot syndrome and depression which are demonstrated to be the predictor of disability related to activities of daily living. ${ }^{2.3}$

The clinical importance of DMII have been increased due to a dramatical increase of its incidence together with high life expectancy. In Saudi Arabia, the life expectancy has been increased in 2011 to be 76 years (males: 74, females 80 years) as compared to 73.13 years in 2005 (males: 72.24, females 74.41 years). ${ }^{4}$ Additionally the diabetes prevalence is showed to be $30 \%$ of Saudi population $(34.1 \%$ in males and $27.6 \%$ in females). $\frac{5}{-}$

Patients with DMII have reported to be more disabled in self-care tasks and other daily living activities than non-diabetic subjects because of many hand complications. However, there is a limited number of researches related to such problems. $\frac{5}{}$

The longer the duration of illness the greater will be the reduction of hand grip strength, agility, and disabilities. ${ }^{2,6,7}$

Muscle weakness has been associated with DMII can be attributed to increased insulin tissue resistance and hyperglycaemia, which cause a reduction in the number of mitochondria in the muscle cells, a decrease in glycogen synthesis and an increase in the amount of circulating systemic inflammatory cytokines, all of which have a detrimental effect on the skeletal muscles. $\stackrel{8}{ }$

Additionally, the physiological cross section of the muscles is significantly lower in individuals with diabetes, with being worse with longer duration of illness and poorer control. ${ }^{9}$ Diabetic stiff hand syndrome or limited joint mobility syndrome, is found in $8-50 \%$ of all patients with type 1 diabetes and is also seen in type 2 diabetic patients. The prevalence increases with duration of diabetes. This condition is associated with and predictive of other diabetic complications. ${ }^{9}$

The metabolic disturbances associated with DMII also cause damage to the connective tissues of the hand leading to limitation in joint range of motion, Dupuytren's contracture, and flexor tenosynovitis in approximately $50 \%$ of individuals with DMII. $\frac{2,7,9}{2}$ 
Carpel tunnel syndrome has been reported to be the most common pathology with an incidence of $45 \%$. Its specific relationship to diabetes is thought to be median nerve entrapment caused by the diabetesinduced connective tissue changes. ${ }^{7}$

Identification of such changes can be a warning signs for more diabetic complications. To our best knowledge, there are no studies in this theme in Saudi Arabia. Therefore, we tested hypothesis that hand grip and pinch strength are lowered in patients with DMII and the effect of that on the functional abilities of the hand.

\section{Methods:}

This cross-sectional study was carried out in the outpatient clinics of the King Khalid Hospital. The target population of this study consisted of diabetic forty subjects (mean age: $51 \pm 5.58$ years) and Forty matched healthy volunteers (mean age: $49.05 \pm 6.73$ years). The mean duration of illness of the patients in the diabetic

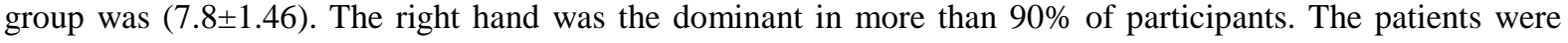
recruited from diabetes's clinic in the king Khalid hospitals, Hail, KSA. Participant with history of cervical spondylosis, cervical pott's disease, primary and metastatic tumours of the cervical vertebrae, fracture and dislocation of the cervical vertebrae, cervical neurofibromatosis ,dejerine-klumpke's paralysis secondary to birth injury, thoracic outlet syndrome and carpal tunnel syndrome, peripheral nerve injury, Amyotrophic lateral sclerosis, and cervical radiculopathy during the previous 6 months were excluded from the study.

Identifying the participants age at time of diagnosis was used to calculate the duration of illness. The healthy Participants had no glucose intolerance, no history of pain and musculoskeletal problems in the shoulder, arm or hand, no documented history of trauma or brachial plexus injury, peripheral nerve injury, Amyotrophic lateral sclerosis ,cervical radiculopathy in the previous 6 months.

Anthropometric variables included body weight, duration of illness, occupation were taken. Body weight was measured using a calibrated scale (GIMA Pegaso Electronic Body Scale-Italy). Duration of illness, occupation were taken through assessment charts. JAMAR Hydraulic hand dynamometer and a pinch gauge (5030J1 ,CANADA) were used to measure the hand muscle strength and the key pinch respectively. Both the dynamometer and pinch gauge were reset to the starting point (zero) before each reading as the red peak-hold needle was rotated counter-clockwise to zero. Subjects seated comfortably on a chair with armrests. The shoulder was adducted and rotated neutrally, with 90 degrees elbow flexion, and the forearm and wrist in a neutral position. Measurements were started with the dominant hand. A proper verbal support ("press the handle/button as could as possible") was used during the measurements the peak-hold needle then automatically recorded the maximum force was exerted. The Reading is then recorded.

\section{Statistical analysis:}

The Social Package for Social Sciences (SPSS) version 16.0 (SPSS Inc, Chicago, IL, USA) was used to analyze the data. Descriptive statistics such as means, standard deviations were used to describe the participants' demographic data. Statistical measures of the mean scores and standard deviation were calculated for the baseline measurement for each participant. Paired $t$ test was used for comparing clinical patient's data it was also used for within group comparison of the hand grip and key pinch.

\section{Results}

The anthropometric characteristics and of the subjects were given in Tables 1. There were no significant difference between the groups with respect to age, body weight $(\mathrm{p}>0.05)$. the mean age of the patients represented in $\mathrm{G}_{1}$ is $51 \pm 5.59$ and $49.84 \pm 4.82$ in $\mathrm{G} 2$. The mean body weight in $\mathrm{G}_{1}$ is $82.62 \pm 11.14$ and 48.32 \pm 12.01 . Patients with hypertension represented $66.21 \%$ and $52.57 \%$ in the diabetic group the mean duration of illness in the diabetic group is $7.41 \pm 3.76$ years.

Table 1: The anthropometric and clinical characteristics patients and of the healthy subjects

\begin{tabular}{|l|c|l|c|}
\hline & Group 1 & Group 2 & P value \\
\hline Age (years) & $51 \pm 5.59$ & $48.05 \pm 6.73$ & 0.42 \\
\hline Body weight $(\mathrm{Kg})$ & $48.32 \pm 12.01$ & $82.62 \pm 11.14$ & 0.14 \\
\hline Hypertension & $66.21 \%$ & $52.57 \%$ & 0.43 \\
\hline Duration of illness & $7.41 \pm 3.76$ & ---- & --- \\
\hline
\end{tabular}

Handgrip strength was measured with an isometric hand dynamometer and comparisons were made between diabetic and non-diabetic females. The t-test were used to analyze the significance difference in hand grip and pinch power strength between the diabetic and the non- diabetic subjects $(\mathrm{p} \leq 0.05)$. Hand grip strength was significantly lower in the diabetic group as clearly shown in figure 1. 
The mean value of the right hand of the diabetic patient group was $24.9 \pm 5.63 \mathrm{Ib}$ and $42.75 \mathrm{Ib}$ in the healthy subject. The mean value of left hand of the diabetic patients was $19.07 \pm 4.64$ while it is $37.87 \pm 4.65 \mathrm{Ib}$ in the non diabetic subjects.

Pinch power strength was significantly lower in the diabetic group, the mean value of the right hand of the diabetic patient group was $5.75 \pm 1.75 \mathrm{Ib}$ and $11.3 \pm 2.11 \mathrm{Ib}$ in the healthy subject. The mean value of left hand of the diabetic patients was $5.52 . \pm 2.07$ while it is $10.72 \pm 1.15 \mathrm{Ib}$ in the nondiabetic subjects.

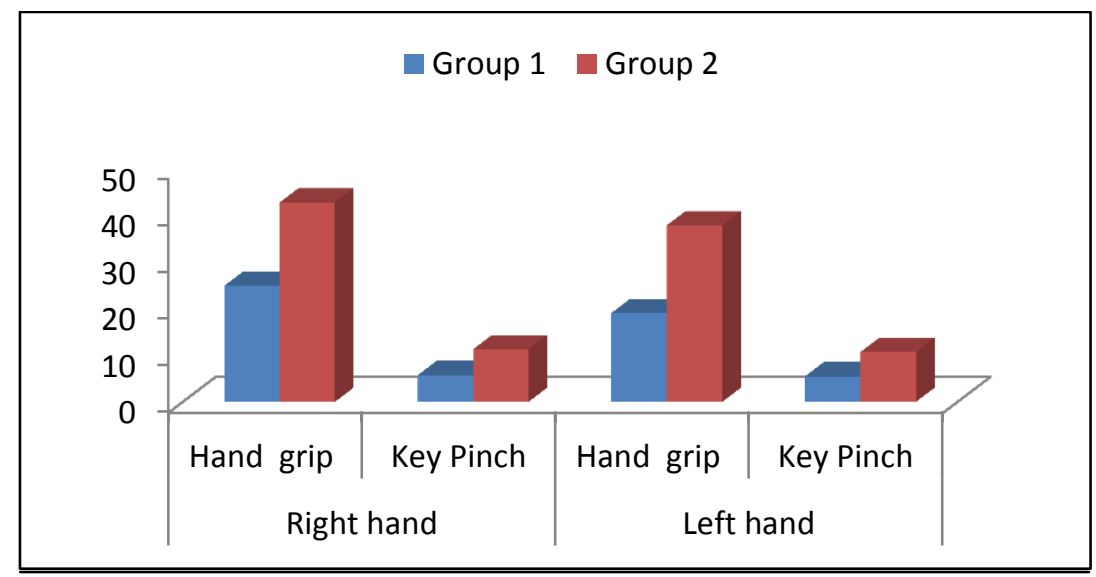

Figure 1. The results of the hand grip strength test (Ib)with the Jamar dynamometer were significantly lower in the diabetic group compared with the control group $(\mathrm{p}<0.05)$. The key pinch strength value for the right hand (Ib) was significantly lower in the diabetic group than the control subject.

\section{Discussion}

Diabetes mellitus is usually associated with mild hand muscle weakness associated with peripheral sensory neuropathy in DM patients..$^{10}$ To the best of our knowledge, this is the first study in Saudi Arabia that measure the influence of diabetes on grip strength and pinch power and emphasize the importance of measuring hand functions as a measure for prevention and treatment of hand complications caused by DM.

The results of the current study revealed that there is a significant decrease of the hand grip strength using the Jamar dynamometer in the diabetic patient compared with healthy matched subjects . Again, the key pinch power value for the right and left hand was markedly decreased in the DM group.

The grip strength test was commonly done to evaluate the performances of hand muscles by measuring the maximal grip force that could be executed in one muscular contraction. ${ }^{10,11}$ the results of this study is not consistent with other researches that find that there was a significant reduction in the muscle strength of the ankle dorsal and plantar flexors using isokinetic dynamometer, but there was insignificant reduction in muscle strength of the wrist flexors and extensors. 11

Our study presented slight difference between the dominant and nondominant hand and this not go with some researcher reported that there were no marked differences in grip strength between the dominant and nondominant hand 28 but go with others who found only a slight, non-significant difference in hand strength between the dominant and non-dominant hand $\underline{.12}$

On the other hand, the results of this study is in close agreement with Ezema and colleagues who stated that DMII seems to result in a decrease in handgrip strength in both male and female adults. This physical limitation may contribute to low productivity in people with DMII. 13

Our results also is consistent with other studies that reported that DMII is associated with poorer upper limb muscle strength and quality. ${ }^{14,15}$ These features may contribute to upper limb functional limitation and physical disability in individuals with long-standing type 2 diabetes. $\frac{16,17}{}$

An important limitation of this study is the small sample size. Nevertheless, we were able to show that there is a significant decrease of the hand grip strength and pinch power in the diabetic patients compared with the normal subjects. Further detailed studies are needed to explain the relationship between neuropathy and hand grip strength and pinch power.

\section{Conclusion}

The results of the current study concluded that the hand muscles strength represented in grip and pinch power which are important parameters of hand function is significantly affected by long standing type 2 diabetes mellitus Individuals with long-standing type 2 diabetes mellitus have been found to have an increased risk of developing functional disabilities due to hand muscle weakness. 


\section{References:}

[1]. Centers for Disease Control and Prevention. National Diabetes Fact Sheet, 2011. Atlanta, GA: Centers for Disease Control and Prevention, US Department of Health and Human Services; 2011. http://www.cdc.gov/diabetes/pubs/pdf/ndfs_2011. pdf. Accessed April 16, 2012

[2]. Savaş S, Köroğlu BK, Koyuncuoğlu HR, Uzar E, Celik H, Tamer NM. The effects of the diabetes related soft tissue hand lesions and the reduced hand strength on functional disability of hand in type 2 diabetic patients. Diabetes Res Clin Pract. 2007 Jul;77(1):7783.

[3]. Bruce DG., Davis WA. Davis T.M. Longitudinal predictors of reduced mobility and physical disability in patients with type 2 diabetes: the Fremantle Diabetes Study, Diab. Care 28 (10) (2005) 2441-2447.

[4]. WHOdatabas2013 http://www.who.int/gho/publications/world_health_statistics/EN_WHS2013.

[5]. Badran M and Laher I. Type II Diabetes Mellitus in Arabic-Speaking Countries. Int J Endocrinol. 2012;2012:902873.

[6]. Mathy SC, Freid LP, Volpato S, Williamson J, Brancati FL, Blaum CS. Patterns of disability related to diabetes mellitus in older women, J. Geontol. A: Biol. Sci. Med. Sci. 2004; 59 (2):148-153.

[7]. Gamstedt A, Holm-Glad J, Ohlson CG, Sundstrom M. Hand abnormalities are strongly associated with the duration of diabetes mellitus. J Intern Med. 1993;234:189-193.

[8]. Helmersson, JB. Larsson VA. Basu S. Association of type 2 diabetes with cyclooxygenase-mediated inflammation and oxidative stress in an elderly population. Circulation 2004, 109: 1729-1734.

[9]. Kim RP, Edelman SV, Kim DD. Musculoskeletal Complications of Diabetes Mellitus. CLINICAL DIABETES 2001, 19(3): 132135.

[10]. Redmond CL, Bain GI, Laslett LL, McNeil JD. Hand syndromes associated with diabetes: impairments and obesity predict disability. J Rheumatol. 2009;36:2766-2771.

[11]. Infante JR, Rosenbloom AL, Silverstein JH, Garzarella L, Pollock BH. Changes in frequency and severity of limited joint mobility in children with type 1 diabetes mellitus between 1976-78 and 1998. J Pediatr 2001;138:33-7.

[12]. Park SW, Goodpaster BH, Strotmeyer ES, de Rekeneire N,Harris TB, Schwartz AV, et al. Decreased muscle strength and quality in older adults with type 2 diabetes: the health,aging, and body composition study. Diabetes 2006;55:1813-8

[13]. Ezema CI, Iwelu EV, Abaraogu UO, Olawale OA. Handgrip Strength in Individuals with Long-Standing Type 2 Diabetes Mellitus: A preliminary report. AJPARS 2012, 4( 1): $67-71$.

[14]. Bus SA, Yang QX., Wang JH, Smith MB, Wunderlich, Cavanagh PR., Intrinsic muscle atrophy and toe deformity in the diabetic neuropathic foot: a magnetic resonance imaging study, Diabetes Care 2002, 25: 1444-1450.

[15]. Andersen H, Poulsen PL, Mogensen CE, Jakobsen J. Isokinetic muscle strength in long-term IDDM patients in relation to diabetic complications, Diabetes 1996, 45: 440-445.

[16]. Zdirenc MO, Biberog LU, zcan AS. Evaluation of physical fitness in patients with type 2 diabetes mellitus, Diabetes Res. Clin. Pract. 2003, 60: 171-176.

[17]. Clerke A, J. Clerke. A literature review of the effects of handedness on isometric grip difference of the left and right hands. American Journal of Occupational Therapy 2001, 55(2): 206-11. 